\title{
Protective Effect of Persimmon (Diospyros kaki) Peel Proanthocyanidin against Oxidative Damage under $\mathrm{H}_{2} \mathrm{O}_{2}$-Induced Cellular Senescence
}

\author{
Young A Lee, ${ }^{a}$ Eun Ju Cho, ${ }^{b}$ and Takako YoKozawA ${ }^{*, a}$ \\ ${ }^{a}$ Institute of Natural Medicine, University of Toyama; 2630 Sugitani, Toyama 930-0194, Japan: and ${ }^{b}$ Department of Food \\ Science and Nutrition, Pusan National University; 30 Jangjeon-dong, Geumjeong-gu, Busan 609-735, Korea. \\ Received November 21, 2007; accepted March 19, 2008
}

\begin{abstract}
8-Hydroxy-2'-deoxyguanosine (8-OHdG), one of the most abundant oxidative DNA adducts, is used as an indicator of oxidative DNA damage associated with aging. Among homologs of the silent information regulator (Sir), sirtuin 1 (SIRT1) is suggested as a regulator of the apoptotic response to DNA damage. Since it has been suggested that the aging process can be delayed by the attenuation of oxidative damage such as DNA damage or SIRT1 modulation, we focused on the protective effect against cellular oxidative damage of persimmon peel, a proanthocyanidin-rich food, in relation to its level of polymerization. We confirmed that 8-OHdG expression in TIG-1 human fibroblasts was increased by treatment with $300 \mu_{\mathrm{M}} \mathrm{H}_{2} \mathrm{O}_{2}$ for $2 \mathrm{~h}$. On the other hand, the nuclear SIRT1 level was decreased in $\mathrm{H}_{2} \mathrm{O}_{2}$-treated as compared with non-pretreated cells. However, pretreatments with polymers and oligomers led to a decrease in 8-OHdG and elevation in nuclear SIRT1 expression in a concentration-dependent manner. In particular, oligomers exerted a stronger effect. The present study supports the protective potential of proanthocyanidin from persimmon peel against oxidative damage under the aging process, and suggests that the polymerization of proanthocyanidin plays an important role in retarding aging in a cellular senescence model.
\end{abstract}

Key words proanthocyanidin; 8-hydroxy-2'-deoxyguanosine; sirtuin 1; $\mathrm{H}_{2} \mathrm{O}_{2}$-induced premature senescence; polymerization; persimmon peel

Aging is an inevitable process in all living organisms. It is generally accepted that molecular oxidative damage induced by reactive oxygen species (ROS) is the main causal factor underlying the aging process and senescence-associated losses in physiological functions. ${ }^{1,2)}$ With the aging process, organisms accumulate persistent DNA lesions with singleand double-stranded breaks. This supports the physiological importance of DNA damage in cellular senescence and aging. $^{3-5}$ ) Since 8 -hydroxy-2'-deoxyguanosine $(8-\mathrm{OHdG})$ is one of the most abundant oxidative DNA adducts, it has been used as an indicator of oxidative DNA damage associated with aging. ${ }^{6-8)}$

DNA damage is linked to the modulation of Saccharomyces cerevisiae chromatin silencing factor Sir2 (Silent information regulator 2) as the main factor determining life span. Sir2 is an NAD-dependent histone deacetylase that suppresses transcription at several genomic loci. It has been implicated in mediating gene silencing, longevity, and genome stability. It is conceivable that poly(ADP-ribosyl)ation by poly (ADP-ribose) polymerase 1 (PARP-1), which is induced by DNA damage, could modulate protein deacetylation by Sir2 via the $\mathrm{NAD}^{+}$/nicotinamide connection. ${ }^{9)}$ In consequence, cells with damaged DNA are not committed to die by apoptosis; this leaves time for DNA repair. Seven mammalian Sir2 homologs, referred to as sirtuin (SIRT) $1-7$, have been identified. ${ }^{10)}$ Since SIRT1 among Sir2 homologs is a nuclear protein and the mammalian homolog most highly related to Sir2, it has been the focus of studies. ${ }^{11)}$ High activity levels of SIRT1 have been suggested to regulate the apoptotic response to DNA damage. ${ }^{12-14)}$ Therefore, the potential supportive role of antioxidants against oxidative DNA damage and the upregulation of Sir2 has been focused on an attempt to delay the aging process.

Polyphenol has attracted much attention as an antiaging agent through attenuation of DNA damage and activation of
Sir2 as well as the human homolog SIRT1. ${ }^{15,16)}$ Persimmon is one of the proanthocyanidin-rich foods with higher contents in peel than in pulp. ${ }^{17,18)}$ Proanthocyanidin is known as a condensed tannin, a part of a specific group of polyphenolic compounds, and it has been reported to exhibit powerful antioxidant activity. ${ }^{19,20)}$ In this study, we disintegrated polymers and oligomers from proanthocyanidin to evaluate functional properties caused by polymerization. In particular, to study their potential as agents to retard aging, we employed a hydrogen peroxide $\left(\mathrm{H}_{2} \mathrm{O}_{2}\right)$-induced cellular senescence model. We evaluated DNA damage by determination of the 8-OHdG level and SIRT1 expression to clarify the effect of proanthocyanidin against oxidative stress caused by cellular senescence.

\section{MATERIALS AND METHODS}

Reagents and Cells Dulbecco's Modified Eagle Medium (DMEM), paraformaldehyde, Triton X-100, and collagen (from bovine achilles tendon) were purchased from Sigma Chemical Co. (St. Louis, MO, U.S.A.). Calcium- and magnesium-free phosphate-buffered saline (PBS) and 4',6-diamidino-2-phenylindole dihydrochloride $n$-hydrate (DAPI) were purchased from Wako Pure Chemical Industries Ltd. (Osaka, Japan). Fetal bovine serum (FBS) and trypsin-EDTA solution were purchased from JRH Biosciences (Lenexa, KS, U.S.A.) and GIBCO (Grand Island, NY, U.S.A.). Normal human lung diploid fibroblasts (TIG-1) were purchased from Health Science Research Resources Bank (Osaka, Japan). Monoclonal anti-8-hydroxy-2'-deoxyguanosine and polyclonal anti-SIRT1 were purchased from the Japan Institute for the Control of Aging (Nikken SEIL Co., Shizuoka, Japan) and Santa Cruz Biotechnology (Santa Cruz, CA, U.S.A.). Alexa Flour 488-conjugated goat anti-mouse IgG and Alexa Flour 568-conjugated goat anti-rabbit IgG were 
purchased from Molecular Probes (Eugene, OR, U.S.A.).

Fractionation of Polymers and Oligomers from Proanthocyanidin of Persimmon Peel A mixture of freshly crushed persimmon peel (green peel, 5-7 cm in diameter, $3 \mathrm{~kg}$ ) and dried green tea leaves $(450 \mathrm{~g})$ in water containing citric acid (240 g) was boiled for $3 \mathrm{~h}$. At this stage, nucleophillic substitution at the $\mathrm{C}-4$ positions of polymeric proanthocyanidin with monomeric tea catechins occurred, and consequently, the polymeric molecules were converted into oligomers. After cooling, insoluble materials were removed by filtration, and the filtrate was directly applied to a Sephabeads SP 825 column $(10 \mathrm{~cm}$ i.d. $\times 45 \mathrm{~cm}$, Mitsubishi Chemical, Co., Japan). Elution with water (41) washed out non-phenolic compounds consisting of citric acid, sugars, minerals, amino acids, etc. Further elution with water containing increasing amounts of ethanol (20-80\% ethanol, $20 \%$ stepwise elution, 21 each) yielded a mixture of oligomeric proanthocyanidin and tea catechins $(72.2 \mathrm{~g})$. The mixture was subsequently subjected to Sephadex LH-20 column chromatography with ethanol. The monomeric tea catechins were eluted out with ethanol, and further elution with $50 \%$ aqueous acetone yielded oligomers (51.5 g). The degree of oligomeric polymerization was estimated as 3.3 by quantitative HPLC analysis of thiol degradation products, ${ }^{21)}$ while the unit ratios of epigallocatechin (EGC), epicatechin (EC), epigallocatechin 3-O-gallate (EGCg), and epicatechin 3-Ogallate $(\mathrm{ECg})$ in oligomers were determined as $47,15,31$, and $6 \%$, respectively. The preparation of polymeric proanthocyanidin from persimmon was as follows: an aqueous acetone extract of fresh persimmon peel $(500 \mathrm{~g})$ was concentrated, and the resulting insoluble precipitates were removed by filtration. The filtrate was subjected to MCI-gel CHP 20P (Mitsubishi Chemicals, Co., Japan) column chromatography with water containing methanol $(0-80 \%, 20 \%$ stepwise elution) to give polymers $(6.93 \mathrm{~g})$.

Cell Culture and $\mathrm{H}_{2} \mathrm{O}_{2}$ Treatment TIG-1 cells were cultivated in 100-mm culture dishes containing DMEM supplemented with $10 \% \mathrm{FBS}$ at $37^{\circ} \mathrm{C}$ in a humidified atmosphere of $5 \% \mathrm{CO}_{2}$ in air. The cells were detached from the dish by $0.05 \%$ trypsin-EDTA in PBS and subcultured in 8 well chamber slides (Falcon, Franklin Lakes, NJ, U.S.A.) coated with collagen $(10 \mu \mathrm{g} / \mathrm{ml})$. The density and populationdoubling level (PDL) of TIG-1 cells were $1.0 \times 10^{4}$ cells $/ \mathrm{ml}$ and 34, respectively. After incubation for $24 \mathrm{~h}$, cells were treated with polymers and oligomers at concentrations of 1 , 10 , and $20 \mu \mathrm{g} / \mathrm{ml}$. After that, the medium was replaced with a fresh one which contained $300 \mu \mathrm{M} \mathrm{H}_{2} \mathrm{O}_{2}$, and cells were incubated for $2 \mathrm{~h}$.

Immunostaining for 8-OHdG The slices were fixed in $4 \%$ paraformaldehyde and treated with RNase $(100 \mu \mathrm{g} / \mathrm{ml})$ in Tris buffer (10 mM Tri-HCl (pH 7.5), 1 mm EDTA, and $0.4 \mathrm{M}$ $\mathrm{NaCl})$ at $37^{\circ} \mathrm{C}$ for $1 \mathrm{~h}$. After rinsing with PBS, cells were treated with proteinase $\mathrm{K}(10 \mu \mathrm{g} / \mathrm{ml})$ at room temperature for $7 \mathrm{~min}$. After rinsing with PBS, DNA was denatured by treatment with $4 \mathrm{~N} \mathrm{HCl}$ for $7 \mathrm{~min}$ at room temperature. The $\mathrm{pH}$ was adjusted with $50 \mathrm{~mm}$ Tris- $\mathrm{HCl}$ for $5 \mathrm{~min}$ at room temperature. ${ }^{22)}$ After rinsing with $0.2 \%$ Triton $\mathrm{X}$ in PBS, the cells were stained with a monoclonal anti-8-OHdG $(1: 50)$. Alexa Fluor 488-conjugated goat anti-mouse IgG $(1: 200)$ was used as the secondary antibody. The slices were mounted with Aqua-Poly/Mount (Polysciences, Inc., PA, U.S.A.). Fluores- cence images were captured using a fluorescence microscope (AX-80, Olympus, Tokyo, Japan), and 16-19 images per treatment were obtained. The positive expression of $8-\mathrm{OHdG}$ from $160-285$ cells was measured using an image analyzer ATTO densitograph (ATTO, Tokyo, Japan).

Immunostaining for SIRT1 The slices were fixed in 4\% paraformaldehyde and then cells were stained with polyclonal anti-SIRT1 (1:100). Alexa Fluor 568-conjugated goat anti-rabbit IgG $(1: 200)$ was used as the secondary antibody. The slides were mounted with Aqua-Poly/Mount. Fluorescence images were captured using a fluorescence microscope (AX-80, Olympus, Tokyo, Japan), and 16-20 images per treatment were obtained. The positive expression of SIRT1 from 160-300 cells was measured using an image analyzer ATTO densitograph (ATTO, Tokyo, Japan).

DAPI Staining The cells immunostained for $8-\mathrm{OHdG}$ or SIRT1 were rinsed and then stained with DAPI $(2 \mu \mathrm{g} / \mathrm{ml})$ for 10 min. After rinsing with PBS, the slices were mounted with Aqua-Poly/Mount. Fluorescence images were captured using a fluorescence microscope (AX-80, Olympus, Tokyo, Japan) and merged with $8-\mathrm{OHdG}$ or SIRT1.

Statistical Analysis Statistical comparisons were made by one-way analysis of variance followed post hoc by Dunnett's test. Values of $p<0.05$ were considered significant. The means of the data are presented together with the S.E.M.

\section{RESULTS}

Effect of Polymers and Oligomers on 8-OHdG Expression in the $\mathrm{H}_{2} \mathrm{O}_{2}$-Induced Cellular Senescence Model Oxidative DNA damage in TIG-1 cells was evaluated by immunofluorescence detection of $8-\mathrm{OHdG}$. We confirmed that $8-\mathrm{OHdG}$ was specifically expressed in TIG-1 cells by colocalization of the DAPI-positive and 8-OHdG-positive signals in the nucleus (Fig. 1A). Figure 1B shows that $8-\mathrm{OHdG}$ expression was increased by treatment with $300 \mu \mathrm{M} \mathrm{H}_{2} \mathrm{O}_{2}$ for $2 \mathrm{~h}$ ( $189 \%$ of control). However, pretreatments with polymers and oligomers attenuated this increase in 8-OHdG dose-dependently. In particular, oligomers decreased it significantly even at a low concentration of $1 \mu \mathrm{g} / \mathrm{ml}$. At a dose of 20 $\mu \mathrm{g} / \mathrm{ml}$, the $8-\mathrm{OHdG}$ levels were decreased from $189 \%$ to $125 \%$ and $148 \%$ by oligomers and polymers, respectively.

Effect of Polymers and Oligomers on SIRT1 Expression in the $\mathrm{H}_{2} \mathrm{O}_{2}$-Induced Cellular Senescence Model Figure 2 shows the effect of proanthocyanidin on SIRT1 expression in the TIG-1 cellular senescence. The nuclear SIRT1 level was decreased in $\mathrm{H}_{2} \mathrm{O}_{2}$-treated cells as compared with non-pretreated cells, while pretreatments with polymers and oligomers significantly increased the nuclear SIRT1 expression level. In particular, oligomers exerted a stronger effect on the expression of SIRT1 than polymers.

\section{DISCUSSION}

One of the manifestations of aging is the accumulation of damage at both cellular and organismal levels. This damage is initiated by endogenous and exogenous toxicants. Therefore, oxidants such as $\mathrm{H}_{2} \mathrm{O}_{2}$, tert-butylhydroperoxide, hyperoxia, and UV have been employed in in vitro models of aging. ${ }^{23,24)}$ It has been well established that premature senescence is induced in the early passage of young human diploid 

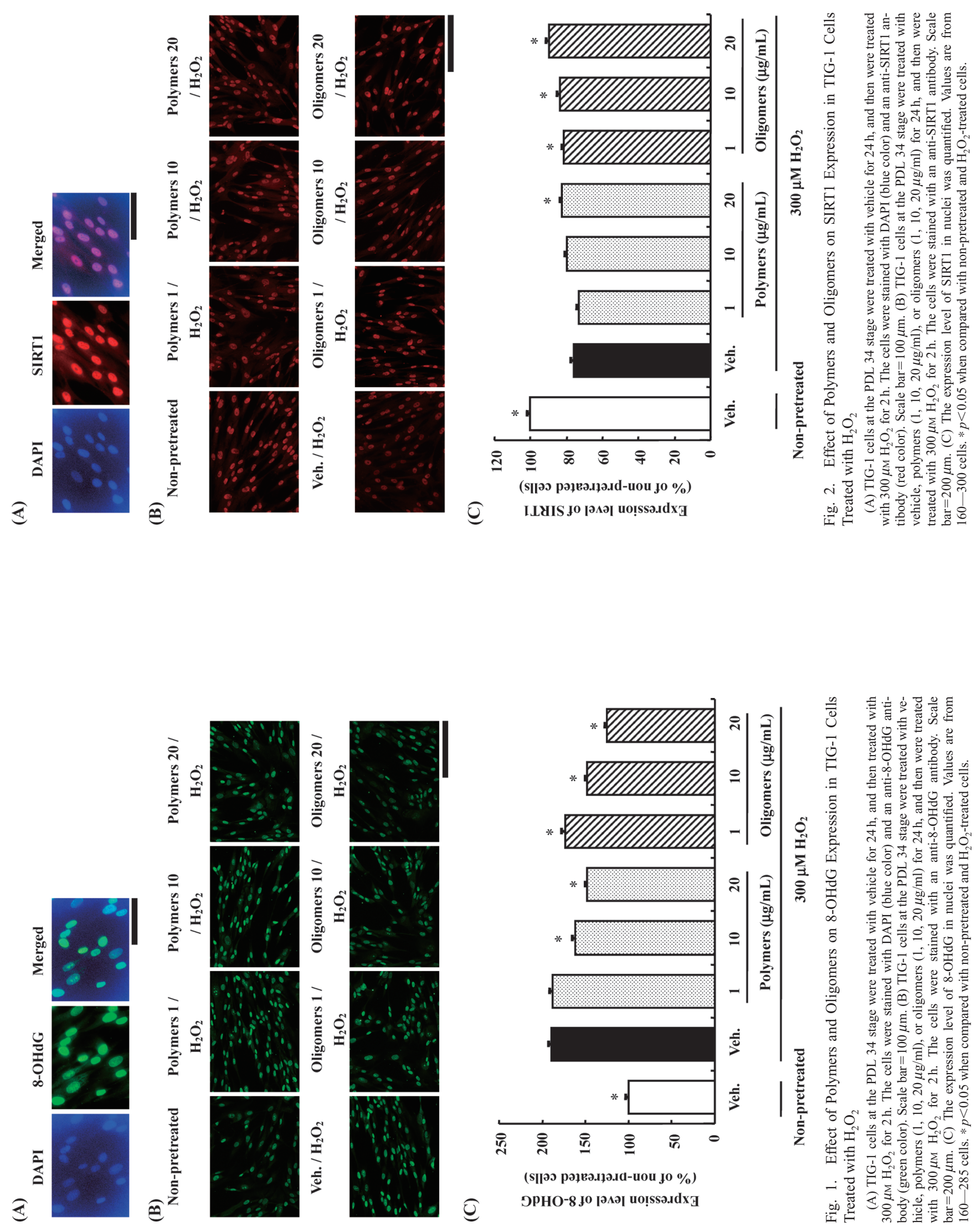
fibroblast by $\mathrm{H}_{2} \mathrm{O}_{2}$; eventually, the cells cannot replicate when stimulated with serum or growth factors, and the senescent cells develop multiple features of replicative senescent cells such as senescence-associated $\beta$-galactosidase activity, $\mathrm{G}_{0} / \mathrm{G}_{1}$ cell cycle arrest, and telomere shortening. ${ }^{8,23,25,26)}$ Therefore, an aging model of $\mathrm{H}_{2} \mathrm{O}_{2}$-induced cellular senescence was employed to investigate the protective potential of proanthocyanidin from persimmon peel against oxidative damage in the nucleus.

DNA damage is an important determinant in the pathophysiology of aging; in fact, the levels of oxidative DNA damage are significantly increased in senescent cells, especially in post-mitotic tissues. 8-Hydroxy- or 8-oxo-deoxyguanosine is recognized as a useful marker for the estimation of DNA damage produced by oxygen radicals endogenously or exogenously. Although numerous oxidative lesions occur in DNA, oxidation of C8 of guanine is one of the more abundant types; it is a major mutagenic lesion producing predominately $\mathrm{G} \rightarrow \mathrm{T}$ transversion mutations. ${ }^{22,27)}$ Accordingly, many researchers have evaluated the $8-\mathrm{OHdG}$ level as a marker of aging under in vivo conditions and in various cell types. ${ }^{6-8)}$ The present results showed the elevation of DNA damage under premature cellular senescence induced by $\mathrm{H}_{2} \mathrm{O}_{2}$. Several studies also demonstrated that $\mathrm{H}_{2} \mathrm{O}_{2}$ treatment of human diploid fibroblasts led to a loss of replicative capacity and/or premature senescence through p53 and Rb pathways by DNA damage. ${ }^{26,28)}$ On the other hand, a decline in the $8-\mathrm{OHdG}$ level was observed by polymers and oligomers pretreatment, suggesting that proanthocyanidin would modulate oxidative DNA damage under $\mathrm{H}_{2} \mathrm{O}_{2}$-induced cellular senescence. In particular, oligomers exerted a stronger protective effect than polymers. Although proanthocyanidin is considered a powerful antioxidant, the relation with its polymerization has not been clarified. Our previous study supported that oligomers, with a lower polymerization level compared to polymers, showed a stronger antioxidative activity. ${ }^{29)}$ The present results also demonstrated that oligomers more effectively attenuated DNA damage by $\mathrm{H}_{2} \mathrm{O}_{2}$ treatment than polymers. Lower-level polymerization plays an important role in the biological activity of proanthocyanidin.

Yeast Sir2 is a heterochromatin component that silences transcription at silent mating loci, telomeres, and ribosomal DNA (rDNA) ${ }^{30)}$ In addition, it suppresses recombination in rDNA and modulates the longevity of most organisms including mammals. ${ }^{31,32)}$ Among the seven mammalian homologues, SIRT1 is the human orthologue of yeast Sir2 and the best-characterized member of mammalian sirtuins. In this study, we clarified that SIRT1 was decreased by $\mathrm{H}_{2} \mathrm{O}_{2}$-induced cellular senescence. Moreover, this change was attenuated by pretreatment with proanthocyanidin, particularly the oligomeric form. Recently, several researchers suggested that interaction between SIRT1 and PARP-1 can markedly deplete intracellular NAD pools following DNA damage. SIRT1 is associated with $\mathrm{NAD}^{+}$concentration induced by PARP activation for the repair of DNA damage. Accordingly, regulation by Sir2 deacetylation of the expression of genes related to apoptosis or longevity may depend on PARP-1 activity. ${ }^{9}$,13) The impairment of SIRT1 under PARP-1 activation induced by DNA damage might promote p53, FOXO, and Bax activities, with sensitization to apoptosis. ${ }^{33)}$
In this study, $\mathrm{H}_{2} \mathrm{O}_{2}$-treated TIG-1 cells showed a higher expression of 8-OHdG and lower expression of SIRT1 levels than non-pretreated TIG-1 cells. Treatment with proanthocyanidin led to a decrease in $8-\mathrm{OHdG}$ and elevation in SIRT1 expression, suggesting that it would have a beneficial effect on DNA damage and longevity. In particular, we showed that polymerization played a crucial role in its activity. Stimulation of SIRT1 is strongly affected by polyphenol stability and metabolism. Oligomers containing dimers, trimers, and tetramers of $\mathrm{EGC}, \mathrm{EC}, \mathrm{EGCg}$, and $\mathrm{ECg}$ are considered to exert a stronger activity than polymers. De Boer et al. ${ }^{16)}$ demonstrated that ECg and EGCg stimulated SIRT1 more effectively than EC and EGC, since these contained gallic acid groups necessary for stimulating the activity of catechins. It was suggested that galloyl and catechol groups are essential for DNA binding. ${ }^{34-36)}$ Therefore, the structural difference between oligomers and polymers is considered to be an important factor regarding proanthocyanidin's action. Proanthocyanidin differs from all other natural polyphenols by virtue of its polymeric nature. It is made of flavan-3-ol units and its average degree of polymerization generally varies between 3 and 11. The biological effect differs in relation to its utilization in biological systems. It is absorbed through the gut barrier, and its absorption depends on the degree of polymerization. Low-molecular-weight proanthocyanidins are known as sustained-release antioxidants; on the contrary, high-molecular-weight proanthocyanidins can exert their antioxidant activity in the digestive tract and protect lipids, proteins, and carbohydrates from oxidative damage during digestion and spare soluble antioxidants. ${ }^{37)}$ The present study demonstrated that the oligomeric form of proanthocyanidin was more effective in protecting against DNA damage by aging and to induce SIRT expression than the polymeric form. With consideration of absorption in biological systems and functional properties, oligomers rather than polymers from proanthocyanidin of persimmon peel are expected to be developed as a promising anti-aging agent.

Proanthocyanidin has been reported to exhibit various biological effects such as protection against cancer and cardiovascular disease, despite its high-molecular-weight possibly causing limited absorption into the intracellular matrix. ${ }^{38)}$ Recently, it was reported that proanthocyanidin may bind to cell surface proteins such as integrins and lamin receptors. ${ }^{39}$ ) This may lead to intracellular signaling into nuclear through stress-activated signaling, include mitogen-activated protein kinase. ${ }^{40)}$ Therefore, we focused on the possibility that polymeric and oligomeric proanthocyanidin can regulate intracellular signaling regardless of the lower absorption rate. In particular, oligomeric proanthocyanidin may have an improved absorption rate, because it is reported that dimers and trimers of proanthocyanidin can be absorbed on to epitherial cells such as Caco-2 cells. ${ }^{41)}$ Accordingly, oligomeric proanthocyanidin may act more effectively on the cellular system than polymeric proanthocyanidin via a direct and/or indirect action. However, further studies are needed to elucidate the difference in biological action by oligomerizations.

In the study, we clarified the protective effect of proanthocyanidin from persimmon peel against DNA damage and SIRT expression in the aging process. In particular, oligomers were more protective than polymers under conditions of $\mathrm{H}_{2} \mathrm{O}_{2}$-induced premature senescence. This suggests 
that the polymerization of proanthocyanidin plays an important role to retard aging in cellular senescence model.

Acknowledgements We wish to thank Dr. Chihiro Tohda for helpful comments. This work was supported in part by the Grants-in-Aid (C) from the Ministry of Education, Culture, Sports, Science, and Technology, Japan (No. 19500661 to T.Y.).

\section{REFERENCES}

1) Beckman K. B., Ames B. N., Physiol. Rev., 78, 547-581 (1998).

2) Sohal R. S., Mockett R. J., Orr W. C., Free Radic. Biol. Med., 33, 575-586 (2002).

3) Barnett Y. A., King C. M., Mutat. Res., 338, 115-128 (1995).

4) Kawanishi S., Hiraku Y., Oikawa S., Mutat. Res., 488, 65-76 (2001).

5) Chen J. H., Ozanne S. E., Hales C. N., DNA Repair (Amst.), 4, 11401148 (2005).

6) Fraga C. G., Shigenaga M. K., Park J. W., Degan P., Ames B. N., Proc. Natl. Acad. Sci. U.S.A., 87, 4533-4537 (1990).

7) Hamilton M. L., Van Remmen H., Drake J. A., Yang H., Guo Z. M., Kewitt K., Walter C. A., Richardson A., Proc. Natl. Acad. Sci. U.S.A., 98, 10469-10474 (2001).

8) Wolf F. I., Torsello A., Covacci V., Fasanella S., Montanari M., Boninsegna A., Cittadini A., Exp. Gerontol., 37, 647-656 (2002).

9) Zhang J., Bioessays, 25, 808-814 (2003).

10) Frye R. A., Biochem. Biophys. Res. Commun., 273, 793-798 (2000).

11) Frye R. A., Biochem. Biophys. Res. Commun., 260, 273-279 (1999).

12) Chen W. Y., Wang D. H., Yen R. C., Luo J., Gu W., Baylin S. B., Cell, 123, 437-448 (2005).

13) Kruszewski M., Szumiel I., DNA Repair (Amst.), 4, 1306-1313 (2005).

14) Wang C., Chen L., Hou X., Li Z., Kabra N., Ma Y., Nemoto S., Finkel T., Gu W., Cress W. D., Chen J., Nat. Cell Biol., 8, 1025-1031 (2006).

15) Borra M. T., Smith B. C., Denu J. M., J. Biol. Chem., 280, 1718717195 (2005).

16) de Boer V. C., de Goffau M. C., Arts I. C., Hollman P. C., Keijer J., Mech. Ageing Dev., 127, 618 -627 (2006).

17) Gorinstein S., Zemser M., Weisz M., Halevy S., Deutsch J., Tilis K., Feintuch D., Guerra N., Fishman M., Bartnikowska E., Biosci. Biotechnol. Biochem., 58, 1087-1092 (1994).
18) Gorinstein S., Zachwieja Z., Folta M., Barton H., Piotrowicz J., Zemser M., Weisz M., Trakhtenberg S., Martin-Belloso O., J. Agric. Food Chem., 49, 952-957 (2001).

19) Dixon R. A., Xie D. Y., Sharma S. B., New Phytol., 165, 9-28 (2005).

20) Xie D. Y., Dixon R. A., Phytochemistry, 66, 2127-2144 (2005).

21) Tanaka T., Takahashi R., Kouno I., Nonaka G., J. Chem. Soc., Perkin. Trans. 1, 3013-3022 (1994).

22) Yarborough A., Zhang Y. J., Hsu T. M., Santella R. M., Cancer Res., 56, 683-688 (1996).

23) Dumont P., Burton M., Chen Q. M., Gonos E. S., Frippiat C., Mazarati J. B., Eliaers F., Remacle J., Toussaint O., Free Radic. Biol. Med., 28 , $361-373(2000)$.

24) Toussaint O., Royer V., Salmon M., Remacle J., Biochem. Pharmacol., 64, 1007-1009 (2002)

25) Chen Q., Ames B. N., Proc. Natl. Acad. Sci. U.S.A., 91, 4130- 4134 (1994).

26) Duan J., Duan J., Zhang Z., Tong T., Int. J. Biochem. Cell Biol., 37, 1407-1420 (2005).

27) Kasai H., Mutat. Res., 387, 147-163 (1997)

28) Lou Z., Chen J., Exp. Cell Res., 312, 2641-2646 (2006)

29) Lee Y. A., Cho E. J., Tanaka T., Yokozawa T., J. Nutr. Sci. Vitaminol., 53, 287-292 (2007).

30) Imai S., Armstrong C. M., Kaeberlein M., Guarente L., Nature, 403 , $795-800(2000)$

31) Guarente L., Genes Dev., 14, 1021-1026 (2000).

32) Michan S., Sinclair D., Biochem. J., 404, 1-13 (2007).

33) Porcu M., Chiarugi A., Trends Pharmacol. Sci., 26, 94-103 (2005).

34) Kanazawa K., Uehara M., Yanagitani H., Hashimoto T., Arch. Biochem. Biophys., 455, 197-203 (2006).

35) Kuzuhara T., Sei Y., Yamaguchi K., Suganuma M., Fujiki H., J. Biol. Chem., 281, 17446-17456 (2006).

36) Kuzuhara T., Tanabe A., Sei Y., Yamaguchi K., Suganuma M., Fujiki H., Mol. Carcinog., 46, 640-645 (2007).

37) Bagchi D., Bagchi M., Stohs S. J., Das D. K., Ray S. D., Kuszynski C. A., Joshi S. S., Pruess H. G., Toxicology, 148, 187-197 (2000).

38) Santos-Buelga C., Scalbert A., J. Sci. Food Agric., 80, 1094-1117 (2000).

39) Kondo K., Uchida R., Tokutake S., Maitani T., Bioorg. Med. Chem., 14, 641-649 (2006).

40) Slack B. E., Siniaia M. S., J. Cell Biochem., 95, 366-378 (2005).

41) Deprez S., Mila I., Huneau J. F., Tome D., Scalbert A., Antioxid. Redox Signal., 3, 957-967 (2001). 